\title{
Algebraic determination of limit cycles in a family of three-dimensional piecewise linear differential systems ${ }^{\text {th }}$
}

\author{
Jaume Llibre ${ }^{\mathrm{a}}$, Enrique Ponce*,b, Javier Ros ${ }^{\mathrm{b}}$ \\ ${ }^{a}$ Departament de Matemàtiques, Universitat Autònoma de Barcelona, 08193 Bellaterra, \\ Barcelona, Spain. \\ ${ }^{b}$ Departamento Matemática Aplicada II. Universidad de Sevilla. E.T.S. Ingenieros. \\ Camino de los Descubrimientos, 41092-SEVILLA, Spain.
}

\begin{abstract}
We study a one-parameter family of symmetric piecewise linear differential systems in $\mathbb{R}^{3}$ which is relevant in control theory. The family, which has some intersection points with the adimensional family of Chua's circuits, exhibits more than one attractor even when the two matrices defining its dynamics in each zone are stable, in an apparent contradiction with the 3dimensional Kalman's conjecture.
\end{abstract}

For these systems we characterize algebraically their symmetric periodic orbits and obtain a partial view of the one-parameter unfolding of its triplezero degeneracy. Having at our disposal exact information about periodic orbits of a family of nonlinear systems, which is rather unusual, the analysis allows us to assess the accuracy of the corresponding harmonic balance

\footnotetext{
«The first author is partially supported by a MEC/FEDER grant number MTM200803437 and by a CICYT grant number 2009SGR 410. The second and third authors are partially supported by a MEC/FEDER grant number MTM2009-07849. E. Ponce acknowledges the hospitality and support from Centre de Recerca Matemàtica, Bellaterra, Barcelona, Spain, from January to July 2007.

${ }^{*}$ Corresponding author

Email addresses: jllibre@mat.uab.cat (Jaume Llibre), eponcem@us.es (Enrique Ponce), javieros@us.es (Javier Ros)
} 
predictions. Also, it is shown that certain conditions in Kalman's conjecture can be violated without losing the global asymptotic stability of the origin. Key words: Limit cycles, periodic orbits, Kalman's conjecture, harmonic balance, piecewise linear differential systems

\section{Introduction and statement of the main results}

In control theory (see $[1,2,3,4,5])$ an important class of differential systems are the Lur'e systems

$$
\dot{\mathbf{x}}(t)=\mathbf{A x}(t)+\mathbf{b} \xi(t), \quad \sigma(t)=\mathbf{c}^{T} \mathbf{x}(t),
$$

where $\mathbf{A}$ is a $n \times n$ constant matrix, $\mathbf{b}, \mathbf{c} \in \mathbb{R}^{n}$, and the input $\xi(t)=\varphi(\sigma(t))$ is the feedback of the output $\sigma$ through a nonlinear continuous function $\varphi: \mathbb{R} \rightarrow \mathbb{R}$. Typically $\varphi(0)=0$, so the origin is an equilibrium point. One of the main problems in this theory is to characterize when the origin is a global attractor. Related to this problem there is the Kalman's conjecture [6] which states that if $k_{1} \leq \varphi^{\prime}(\sigma) \leq k_{2}$ and the linear systems $\dot{\mathbf{x}}(t)=\left(\mathbf{A}+k \mathbf{b c}^{T}\right) \mathbf{x}(t)$ have the origin as a global attractor for all $k \in\left[k_{1}, k_{2}\right]$, then the system (1) has also the origin as a global attractor. Kalman's conjecture is true for dimension $n \leq 3$ and fails for $n>3$, see $[7,8]$.

We restrict our attention to systems $(1)$ in $\mathbb{R}^{3}$, so that we can write $\mathbf{x}(t)=$ $(x(t), y(t), z(t)) \in \mathbb{R}^{3}$, and without loss of generality we assume that $\mathbf{c}=$ $(1,0,0)^{T}$. A common case in applications is when the nonlinear characteristic function is the saturation

$$
\varphi(\sigma)=\sigma \text { for }|\sigma| \leq 1, \quad \varphi(\sigma)=\operatorname{sgn}(\sigma) \text { for }|\sigma|>1
$$


coming from the piecewise linear modeling of the saturation in actuators or some other devices. Thus the function $\varphi$ induces a partition of $\mathbb{R}^{3}$ into three zones $S_{-}, S_{0}$ and $S_{+}$separated by the two planes $\Gamma_{-}$and $\Gamma_{+}$, where $S_{0}=\left\{(x, y, z) \in \mathbb{R}^{3}:-1<x<1\right\}, S_{ \pm}=\left\{(x, y, z) \in \mathbb{R}^{3}: \pm x>1\right\}$, and $\Gamma_{ \pm}=\left\{(x, y, z) \in \mathbb{R}^{3}: x= \pm 1\right\}$. Therefore the differential system (1) splits into the three linear differential systems

$$
\begin{aligned}
& \dot{\mathbf{x}}=\mathbf{A x}-\mathbf{b} \text { in } S_{-} \cup \Gamma_{-}, \\
& \dot{\mathbf{x}}=\mathbf{B x} \quad \text { in } \Gamma_{-} \cup S_{0} \cup \Gamma_{+}, \\
& \dot{\mathbf{x}}=\mathbf{A x}+\mathbf{b} \text { in } S_{+} \cup \Gamma_{+},
\end{aligned}
$$

where $\mathbf{B}=\mathbf{A}+\mathbf{b c}^{T}$.

We remark that these control systems are analytic in $\mathbb{R}^{3} \backslash\left\{\Gamma_{-} \cup \Gamma_{+}\right\}$and only $\mathcal{C}^{0}$ in $\Gamma_{-} \cup \Gamma_{+}$. Since $\mathbf{A x}+\varphi\left(\mathbf{c}^{T} \mathbf{x}\right) \mathbf{b}$ is a Lipschitz function in $\mathbb{R}^{3}$ with Lipschitz constant $L=\max \{\|\mathbf{A}\|,\|\mathbf{B}\|\}$, we obtain from the standard theorems of ordinary differential equations the existence and uniqueness of a solution for any initial conditions $\left(t_{0}, \mathbf{x}_{0}\right) \in \mathbb{R} \times \mathbb{R}^{3}$, and its continuous dependence on initial conditions and parameters. We remark that, in general, the solutions of these piecewise linear control differential systems are $\mathcal{C}^{1}$ but not $\mathcal{C}^{2}$. Note also that if we smooth adequately the characteristic function (2) we can get a monotone smooth characteristic function whose derivative runs through the whole closed interval $[0,1]$.

We recall that a limit cycle is a periodic orbit isolated in the set of all periodic orbits of the differential system, and that a matrix is Hurwitz or stable if it has all the eigenvalues with negative real part. Using matrices A with -1 as a triple eigenvalue and $\mathbf{B}$ with $\lambda<0$ as a triple eigenvalue, 
Moreno y Suárez [9] showed the existence of one stable limit cycle coexisting with the stable equilibrium point at the origin for a unique fixed value of the parameter $\lambda$. Their result was surprising as control practitioners do not expect to find control systems of type (2) having two simultaneous attractors when both matrices $\mathbf{A}$ and $\mathbf{B}$ are stable. Apparently this also seems to be in contradiction with the 3-dimensional Kalman's conjecture. By computing the eigenvalues of the matrix pencil $\mathbf{A}+k \mathbf{b c}^{T}$ for $k \in[0,1]$ one can conclude that there is no such contradiction. Later on, in [10] it was shown numerically that there are two intervals of values of $\lambda$ for which the system studied in [9] has not one but two limit cycles, one stable and the other unstable. One can think that this counter-intuitive coexistence of attractors is related to the triple eigenvalues but this phenomenon is more general as it will be shown in this paper.

Here we will consider system (1) in $\mathbb{R}^{3}$ satisfying (2) and with

$$
\mathbf{A}=\left(\begin{array}{ccc}
-6 & -1 & 0 \\
11 & 0 & -1 \\
-6 & 0 & 0
\end{array}\right), \quad \mathbf{b}_{\mu}=\left(\begin{array}{c}
6(\mu+1) \\
11\left(\mu^{2}-1\right) \\
6\left(\mu^{3}+1\right)
\end{array}\right), \quad \mathbf{c}=\left(\begin{array}{l}
1 \\
0 \\
0
\end{array}\right)
$$

So the eigenvalues of $\mathbf{A}$ are $-1,-2$ and -3 , while the eigenvalues of

$$
\mathbf{B}_{\mu}=\mathbf{A}+\mathbf{b}_{\mu} \mathbf{c}^{T}=\left(\begin{array}{ccc}
6 \mu & -1 & 0 \\
11 \mu^{2} & 0 & -1 \\
6 \mu^{3} & 0 & 0
\end{array}\right)
$$

are $\mu, 2 \mu$ and $3 \mu$. The value $\mu=-1$ should be neglected as then $\mathbf{b}_{\mu}=0$, so that the system becomes purely linear.

The singular points of system (3)-(6) are only the origin $(0,0,0)$ if $\mu<0$, the segment $\{(x, 0,0): x \in[-1,1]\}$ if $\mu=0$, and the origin and the two points 
$\mathbf{e}_{ \pm}= \pm\left(\mu^{3}+1,6 \mu\left(1-\mu^{2}\right), 11 \mu^{2}(1+\mu)\right)$, if $\mu>0$. From the eigenvalues of $\mathbf{A}$ and $\mathbf{B}_{\mu}$ we deduce that the origin of system (3)-(6) is an attractor if $\mu<0$, and a repeller if $\mu>0$, while the two singular points $\mathbf{e}_{ \pm}$always are attractors for $\mu>0$. Therefore at $\mu=0$ we detect a degenerate pitchfork bifurcation of equilibrium points. Note that the situation leads to a triple zero eigenvalue, so that the family of systems under study allows to obtain a specific one-parameter unfolding for such triple-zero bifurcation.

The above eigenvalues have been selected in order to reduce the analytic study of the symmetric periodic orbits to the solving process of a set of polynomial equations. With these values the degree of such polynomials is the lowest possible, but other choices of eigenvalues are possible even they should provide higher degree polynomials and consequently longer computations. In fact such eigenvalue configuration is shown to appear inside the adimensional family of Chua's circuits, see [11] and Section 4.

From (3)-(6) our differential systems are symmetric with respect to the origin of $\mathbb{R}^{3}$, i.e. these systems do not change if we change $\mathbf{x}$ by $-\mathbf{x}$. In other words if $\mathbf{x}(t)$ is a solution of a system (1), then $-\mathbf{x}(t)$ is also a solution. When the orbits corresponding to these two solutions coincide, we say that $\mathbf{x}(t)$ is a symmetric solution. In what follows we shall study the symmetric periodic solutions of the one-parameter family of differential systems (3)-(6).

Since the differential system $\dot{\mathbf{x}}=\mathbf{B}_{\mu} \mathbf{x}$ is linear in its domain of definition $\Gamma_{-} \cup S_{0} \cup \Gamma_{+}$, and has no pure imaginary eigenvalues, system (4) has no periodic orbits. Hence it follows that a symmetric periodic orbit intersects the three zones $S_{-}, S_{0}$ and $S_{+}$. In particular a symmetric periodic orbit has at least two points on the plane $\Gamma_{+}$. So every symmetric periodic orbit 
$(x(t), y(t), z(t))$ can be determined given an initial condition of the form $(x(0), y(0), z(0))=\left(1, y_{0}, z_{0}\right) \in \Gamma_{+}$.

Our main contribution is to provide for the first time in the class of differential systems (3)-(6) an analytical tool, more precisely an algebraic tool, for studying the symmetric periodic orbits. Our algebraic approach is based on the closing equations for symmetric periodic orbits. The use of such closing equations can be traced back to Andronov and coworkers [12] and is also called the Point Transformation method, see for instance [13]. In fact we determine for every value of $\mu \in \mathbb{R}$ all the symmetric periodic orbits having two points in the plane $\Gamma_{+}$, as stated in the next result, which constitutes the main contribution of this paper. The possible existence of symmetric periodic orbits with more than two points in $\Gamma^{+}$and non-symmetric periodic orbits is to be studied elsewhere.

Theorem 1. Regarding symmetric periodic orbits of system (3)-(6) having exactly 2 points in the plane $\Gamma_{+}$the following statements hold.

(a) For $\mu<\mu_{S N 1} \approx-17.350$ there exist two periodic orbits, collapsing at $\mu=\mu_{S N 1}$ to disappear for greater values of the parameter $\mu$. One of these two orbits is stable and the other unstable.

(b) For $\mu_{S N 1}<\mu<\mu_{S N 2} \approx-0.036$ there are no periodic orbits.

(c) For $\mu_{S N 2}<\mu<0$ there are two periodic orbits, collapsing at $\mu=\mu_{S N 2}$ to disappear for lower values of the parameter $\mu$. One of these two orbits is stable and the other unstable.

(d) For $\mu=0$ there is one hyperbolic, stable symmetric periodic orbit pass- 


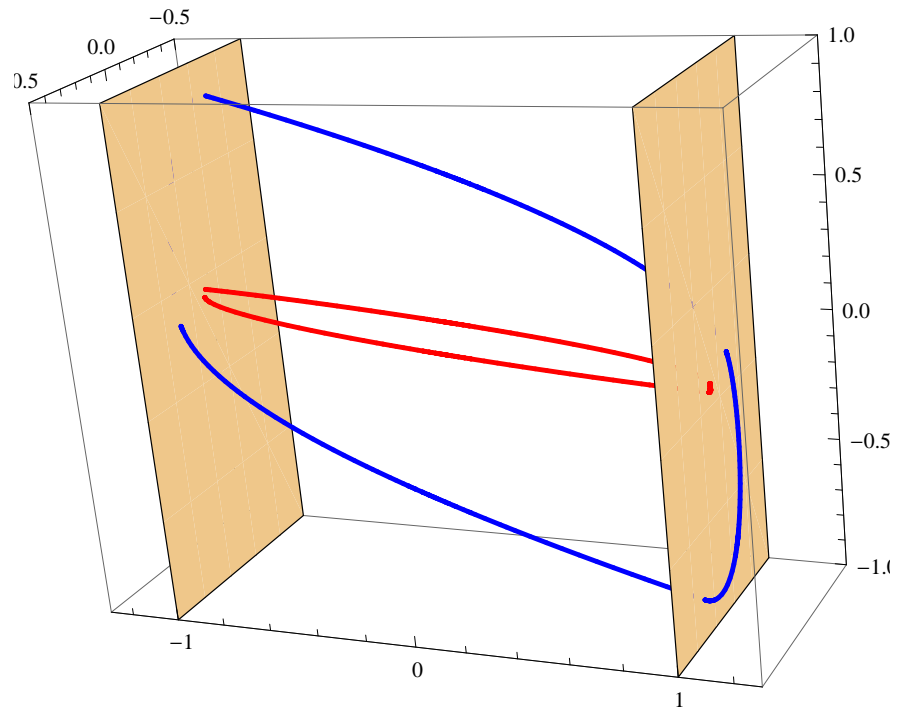

Figure 1: The two symmetric periodic orbits for $\mu=-0.03$. We have also drawn the planes $\Gamma_{+}$and $\Gamma_{-}$. One of the periodic orbits bifurcates from the segment of equilibria $\{(x, 0,0):-1 \leq x \leq 1\}$ when $\mu=0$.

ing through the points $\left(1, y_{0}, z_{0}\right)$ and $\left(1, Y_{0}, Z_{0}\right)$ in $\Gamma^{+}$, where

$$
y_{0}=-(3+2 \sqrt{3}) / 6, \quad Y_{0}=(2 \sqrt{3}-3) / 6, \quad z_{0}=-Z_{0}=\sqrt{3} / 6 .
$$

(e) For $\mu>0$ there is only one periodic orbit, being stable for $\mu<\mu^{*} \approx$ 1.495 and unstable for $\mu>\mu^{*}$.

From Theorem 1 we see that the stated symmetric periodic orbits are organized in two branches which exist for different ranges of parameter $\mu$. Moreover, excepting the three values denoted by $\mu_{S N 1}, \mu_{S N 2}$ and $\mu^{*}$, the periodic orbits are hyperbolic when they exist, and so these periodic orbits will persist under sufficiently small perturbations of $\mathbf{A}, \mathbf{b}_{\mu}, \mathbf{c}$ and $\varphi$. At the critical values $\mu_{S N 1}$ and $\mu_{S N 2}$ system (3)-(6) undergoes a saddle-node 
bifurcation of periodic orbits, that is, two periodic orbit collides to disappear. Note that the numerical approximations for these critical values can be as precise as desired, since they come from solving algebraic equations. This is rather uncommon in practice when dealing with such global bifurcations.

Theorem 1 is proved in Section 2, where algebraic expressions for determining the mentioned branches of symmetric periodic orbits and their stability are provided.

The determination of non-infinitesimal limit cycles is a very difficult problem which usually is solved numerically or by approximate methods, as the harmonic balance method. The harmonic balance method is a tool for estimating periodic orbits by trying to fit a truncated Fourier series choosing the frequency, amplitude and phase so that the lost of precision comes from the neglected harmonics. Under certain hypotheses, the method can exactly detect Hopf bifurcations, see [14]. Thanks to Theorem 1, we have exact information about periodic orbits so that we can assess the accuracy of harmonic balance predictions.

When the Fourier series takes into account only a single sinusoid, the method is also called the describing function method, see for instance [15, $16,17,18]$. This method is widely applied in engineering, because it has a beautiful simple interpretation in terms of intersecting graphs. The describing function method can also be used to analyze the dependence of periodic orbits on the parameters of autonomous systems, obtaining a first approach to the bifurcation diagram associated to the periodic orbits of the system: the so-called first harmonic bifurcation diagram, see [18].

Being an approximate method, the predictions of the first harmonic bi- 
furcation diagram can fail both qualitatively and quantitatively, see [19]. If $\varphi: \mathbb{R} \rightarrow \mathbb{R}$ is an odd piecewise linear function with three pieces, then it is shown in [18] for the nonlinear differential control systems of the form $\dot{\mathbf{x}}(t)=\mathbf{A} \mathbf{x}(t)+\varphi\left(\mathbf{c}^{T} \mathbf{x}(t)\right) \mathbf{b}$ that while in dimension 2 such diagram describes all the qualitative behavior of the periodic orbits, this is not the case in dimension 3. In next result we will obtain the first harmonic bifurcation diagram for differential systems (3)-(6) to be compared with the analytic results of Theorem 1. We will show that such diagram is qualitatively but not quantitatively correct. As a consequence, we also shed some light on the non-equivalence in $\mathbb{R}^{3}$ of Kalman's conditions on the matrix pencil with the global asymptotic stability of the origin, see Remark 1 later.

Proposition 1. Regarding symmetric periodic orbits, the predictions of the describing function method for system (3)-(6) are the following.

(a) For $\mu<\underline{\mu} \approx-16.573$ two periodic orbits are predicted, collapsing at $\mu=\underline{\mu}$ to disappear for greater values of the parameter $\mu$. One of these two orbits is predicted to be stable and the other unstable.

(b) For $\underline{\mu}<\mu<\bar{\mu} \approx-0.0603$ no periodic orbits are predicted.

(c) For $\bar{\mu}<\mu<0$ two periodic orbits are predicted, collapsing at $\mu=\bar{\mu}$ to disappear for lower values of the parameter $\mu$. One of these two orbits is predicted to be stable and the other unstable.

(d) For $\mu \geq 0$ only one periodic orbit is predicted, being stable for $\mu<1$ and unstable for $\mu>1$. 


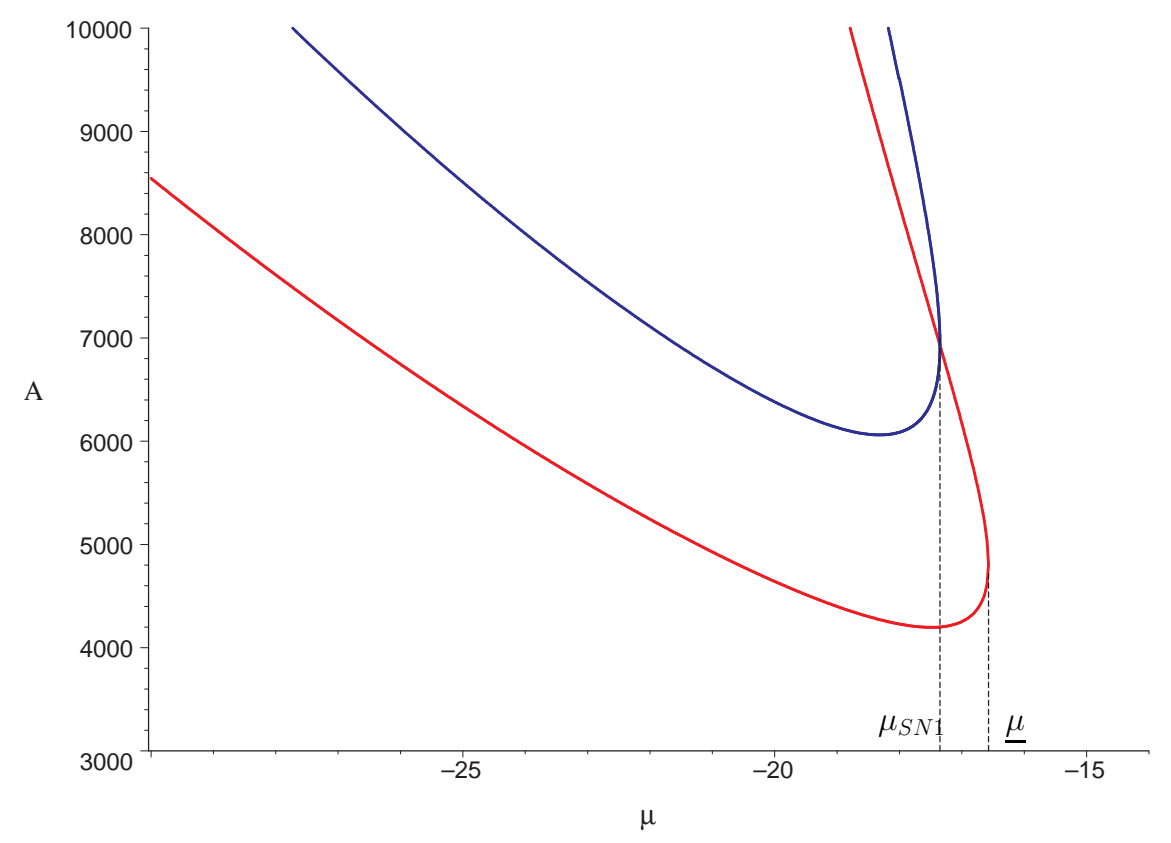

Figure 2: Amplitude of symmetric periodic orbits for big negative values of parameter $\mu$ from the algebraic solutions of the closing equations $\left(A_{C}\right)$ compared with harmonic balance prediction $\left(A_{\mathrm{HB}}\right)$. Note that the harmonic balance predicts the coalescence of two periodic orbits to disappear at a certain critical value $\underline{\mu}$ larger than the actual one $\mu_{S N 1}$.

Proposition 1 is proved in Section 3. In Figs. 2 and 3 we compare the harmonic balance predictions with the exact results provided by Theorem 1 by plotting the amplitude of the different periodic orbits. We have defined $A_{\mathrm{HB}}=\sqrt{1+y_{\mathrm{HB}}^{2}+z_{\mathrm{HB}}^{2}}$, where $\left(1, y_{\mathrm{HB}}, z_{\mathrm{HB}}\right)$ is the point which satisfies $\dot{x}>0$ at the intersection with the plane $x=1$ of the periodic orbit that is predicted by harmonic balance, see [15] and Section 3 for details. Analogously we write $A_{C}=\sqrt{1+y_{0}^{2}+z_{0}^{2}}$, where $\left(1, y_{0}, z_{0}\right)$ is the intersection point (with $\dot{x}>0$ ) of the exact periodic orbit with the plane $x=1$. Such values of $y_{0}, z_{0}$ are obtained from the proof of Theorem 1. Thus in Figs. 2 and 3 the first 


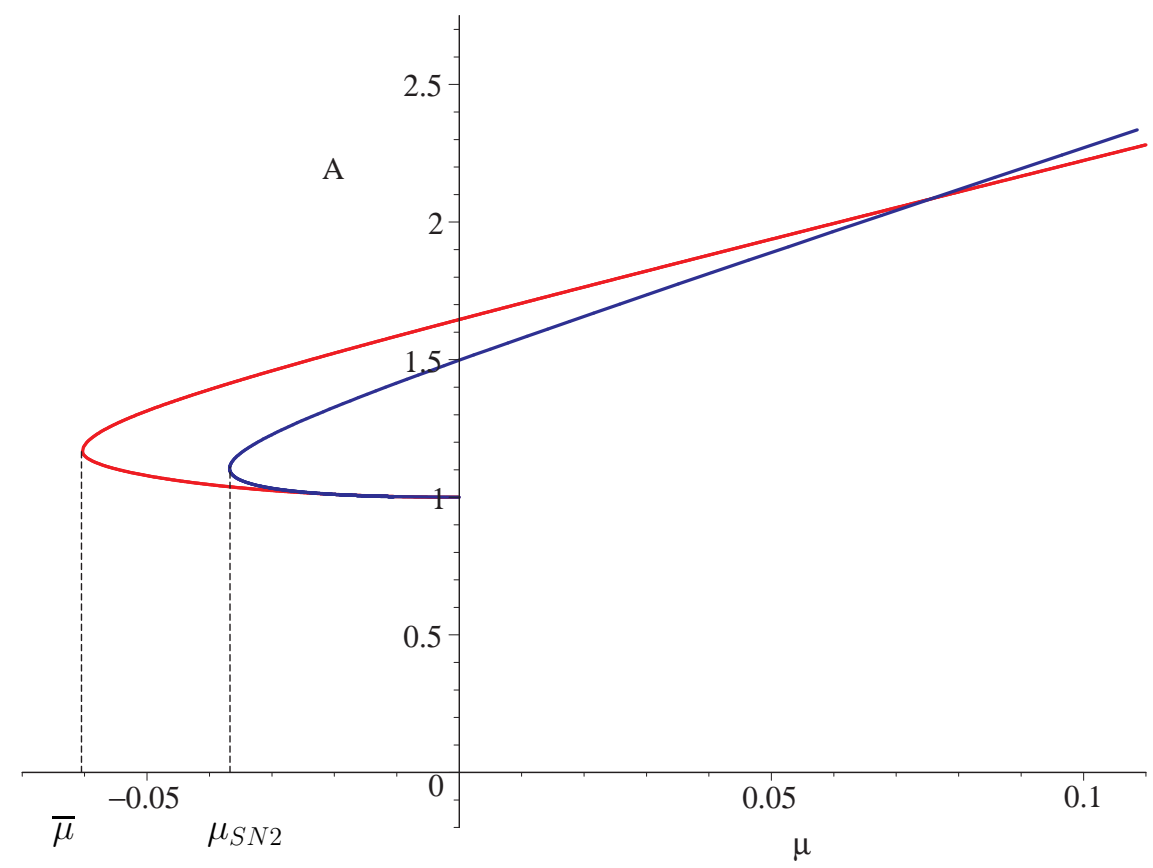

Figure 3: Amplitude of symmetric periodic orbits for small values of parameter $\mu$ from the algebraic solutions of the closing equations $\left(A_{C}\right)$ compared with harmonic balance prediction $\left(A_{\mathrm{HB}}\right)$. Note that now the harmonic balance predicts the coalescence of two periodic orbits to disappear at a certain value $\bar{\mu}$ smaller than the actual one $\mu_{S N 2}$.

harmonic bifurcation diagram for symmetric periodic orbits and their actual bifurcation diagram are plotted. Although the discrepancy between the predictions of the harmonic balance method and the exact values is emphasized in next remark, the method should not be underestimated in detecting the symmetric periodic orbits.

Remark 1. From Theorem 3 of [18] we know that hypotheses of Kalman's conjecture requiring negative real part of the eigenvalues of the matrix pencil are equivalent to the fact that the harmonic balance method does not predict any periodic orbit. Here for the family of systems studied we have detected 
two intervals of the parameter $\mu$, namely $\left(\mu_{S N 1}, \underline{\mu}\right)$ and $\left(\bar{\mu}, \mu_{S N 2}\right)$, where there are not periodic orbits although they are predicted by harmonic balance. This proves that in $\mathbb{R}^{3}$ the failing of the mentioned conditions of Kalman's conjecture is not sufficient to assure the existence of periodic orbits precluding the global asymptotic stability of the origin.

From the above results, see also Fig. 3, we conclude that at $\mu=0$ there is a change in the number of symmetric periodic orbits. In fact, from the proof of Theorem 1 we can assure that one symmetric periodic orbit ends in (or starts from) the segment of equilibrium points that exists at $\mu=0$. On the other hand we already mentioned that at $\mu=0$ there is a degenerate pitchfork bifurcation of equilibrium points. Effectively, for $\mu>0$ there appear two new stable equilibria which are born at the endpoints of the segment of non-isolated equilibrium points that exists for $\mu=0$. This critical value corresponds to the triple zero eigenvalue situation, so that in our one-parameter unfolding for such a bifurcation there are simultaneously both equilibrium points and periodic orbits bifurcations. This one-parameter unfolding of the triple zero bifurcation in piecewise linear systems with symmetry is not yet complete: we conjecture that in this bifurcation there also appear other nonsymmetric periodic orbits for small positive values of $\mu$ associated to the new equilibria. This conjecture, which can be analyzed with the same techniques, is to be investigated elsewhere.

The rest of the paper is organized as follows. In Section 2, as already mentioned, all the algebraic expressions for determining the branches of symmetric periodic orbits leading to prove Theorem 1 are provided. Next, in Section 3 , the computations related to the harmonic balance predictions summarized 
in Proposition 1 are detailed. The intersection points of systems (3)-(6) with the adimensional family of Chua's circuits are shown in Section 4.

\section{Proof of main results}

We start by introducing the algebraic procedure which allows us to convert the problem of determining the symmetric periodic orbits into the problem of solving a set of algebraic equations.

\subsection{The algebraic procedure}

The solution $(x(t), y(t), z(t))$ of system (5)-(6) in the region $S_{+} \cup \Gamma_{+}$ starting at the point $\left(1, y_{0}, z_{0}\right)$ when $t=0$ is

$$
\left(\begin{array}{l}
x(t) \\
y(t) \\
z(t)
\end{array}\right)=\left(\begin{array}{c}
1+\mu^{3} \\
6 \mu\left(1-\mu^{2}\right) \\
11 \mu^{2}(1+\mu)
\end{array}\right)+e^{\mathbf{A} t}\left[\left(\begin{array}{c}
1 \\
y_{0} \\
z_{0}
\end{array}\right)-\left(\begin{array}{c}
1+\mu^{3} \\
6 \mu\left(1-\mu^{2}\right) \\
11 \mu^{2}(1+\mu)
\end{array}\right)\right],
$$

where

$$
e^{\mathbf{A} t}=\frac{u}{2}\left(\begin{array}{ccc}
1-8 u+9 u^{2} & 1-4 u+3 u^{2} & 1-2 u+u^{2} \\
-5+32 u-27 u^{2} & -5+16 u-9 u^{2} & -5+8 u-3 u^{2} \\
6-24 u+18 u^{2} & 6-12 u+6 u^{2} & 6-6 u+2 u^{2}
\end{array}\right),
$$

and $u=e^{-t}$. Note that for all positive values of $t$ we will have $0<u<1$.

Regarding the solution $(X(T), Y(T), Z(T))$ of the system (4)-(6) in the region $\Gamma_{-} \cup S_{0} \cup \Gamma_{+}$starting at the point $\left(1, Y_{0}, Z_{0}\right)$ when $T=0$, it satisfies

$$
(X(T), Y(T), Z(T))^{T}=e^{\mathbf{B}_{\mu} T}\left(1, Y_{0}, Z_{0}\right)^{T} .
$$

The way for computing the symmetric periodic orbits of system (3)(6) having two points in the plane $\Gamma_{+}$is as follows. Assuming that there 
exists one of such periodic orbits, let $\left(1, y_{0}, z_{0}\right) \in \Gamma_{+}$be the point where this periodic orbit enters into the zone $S_{+} \cup \Gamma_{+}$and let $\left(1, Y_{0}, Z_{0}\right) \in \Gamma_{+}$be the point where this periodic orbit exits such zone to enter $S_{0}$. Since this periodic orbit is symmetric it will enter into the zone $S_{-} \cup \Gamma_{-}$through the point $\left(-1,-y_{0},-z_{0}\right) \in \Gamma_{-}$. Let $t$ be the elapsed time for this periodic orbit in going from the point $\left(1, y_{0}, z_{0}\right)$ to the point $\left(1, Y_{0}, Z_{0}\right)$, and let $T$ be the time needed for this periodic orbit to go from the point $\left(1, Y_{0}, Z_{0}\right)$ to the point $\left(-1,-y_{0},-z_{0}\right)$. Then we have the closing equations

$$
(x(t), y(t), z(t))=\left(1, Y_{0}, Z_{0}\right), \quad(X(T), Y(T), Z(T))=\left(-1,-y_{0},-z_{0}\right) .
$$

Equivalently, we can integrate backwards in time the solution from the point $\left(-1,-y_{0},-z_{0}\right)$ to $\left(1, Y_{0}, Z_{0}\right)$ within $S_{0}$, by defining

$$
(\bar{X}(T), \bar{Y}(T), \bar{Z}(T))^{T}=e^{-\mathbf{B}_{\mu} T}\left(-1,-y_{0},-z_{0}\right)^{T} .
$$

We assume in what follows $\mu \neq 0$, since the case $\mu=0$ will be treated in a specific way. Then the exponential $e^{-\mathbf{B}_{\mu} T}$ is the matrix

$$
\frac{v}{2 \mu^{2}}\left(\begin{array}{ccc}
\left(1-8 v+9 v^{2}\right) \mu^{2} & \left(1-4 v+3 v^{2}\right) \mu & 1-2 v+v^{2} \\
\left(-5+32 v-27 v^{2}\right) \mu^{3} & \left(-5+16 v-9 v^{2}\right) \mu^{2} & \left(-5+8 v-3 v^{2}\right) \mu \\
\left(6-24 v+18 v^{2}\right) \mu^{4} & \left(6-12 v+6 v^{2}\right) \mu^{3} & \left(6-6 v+2 v^{2}\right) \mu^{2}
\end{array}\right),
$$

where $v=e^{-\mu T}$. Then we must now have $(\bar{X}(T), \bar{Y}(T), \bar{Z}(T))=\left(1, Y_{0}, Z_{0}\right)$.

If we take $Y_{0}=y(t)$ and $Z_{0}=z(t)$, then the four unknowns $\left(y_{0}, z_{0}, t, T\right)$ associated to this symmetric periodic orbit must satisfy the four equations

$$
x(t)-1=0, \quad 1-\bar{X}(T)=0, \quad y(t)-\bar{Y}(T)=0, \quad z(t)-\bar{Z}(T)=0 .
$$


Using the variables $u=e^{-t}$ and $v=e^{-\mu T}$ instead of $t, T$, equations (10) become

$$
e_{i}\left(y_{0}, z_{0}, u, v\right)=0 \text { for } i=1,2,3,4
$$

being the $e_{i}$ 's polynomials in their variables.

Note that to every symmetric periodic orbit having two points in the plane $\Gamma_{+}$we can associate one solution $\left(y_{0}, z_{0}, u, v\right)$ of equations (11) with $0<u<1$ and $v>1(0<v<1)$ if $\mu<0(\mu>0)$. Also since $\dot{x}=6 \mu-y$ when $x=1$, the two conditions $6 \mu-y_{0}>0$ and $6 \mu-Y_{0}<0$ must be fulfilled. It is not obvious (and not necessarily true in general) the converse statement associating every solution $\left(y_{0}, z_{0}, u, v\right)$ of (11) satisfying the above inequality restrictions to one symmetric periodic orbit. However due to the eigenvalues configuration the following result can be shown.

Proposition 2. Given $\mu \neq 0$, let $\left(y_{0}, z_{0}, u, v\right)$ be one solution of corresponding equations (11) and let $Y_{0}=y(t), Z_{0}=z(t)$ computed from equation (7). Setting $\tilde{y}_{0}=y_{0}-6 \mu$ and $\tilde{Y}_{0}=Y_{0}-6 \mu$, the following statement holds.

If $\mu<0(\mu>0)$ and the four conditions $\tilde{y}_{0}<0, \tilde{Y}_{0}>0,0<u<1$ and $v>1(0<v<1)$ are fulfilled, then system (3)-(6) has a symmetric periodic orbit passing through the points $\left(1, y_{0}, z_{0}\right)$ and $\left(1, Y_{0}, Z_{0}\right)$ of $\Gamma_{+}$.

Proof. Given a solution under the conditions listed, it can be assured that one orbit of system (5) starting at $\left(1, y_{0}, z_{0}\right)$ enters $S_{+}$and after a time $t=-\log u$ arrives at the point $\left(1, Y_{0}, Z_{0}\right)$ to abandon $S_{+}$by entering into $S_{0}$. Similarly we can assure that one orbit of system (4) starting at $\left(1, Y_{0}, Z_{0}\right)$ enters $S_{0}$ and after a time $T=-(\log v) / \mu$ arrives at $\left(-1,-y_{0},-z_{0}\right)$ to abandon $S_{0}$ by entering into $S_{-}$. 
However only if the two quoted orbits do not make unforeseen excursions for intermediate times out of the regions $S_{+}$and $S_{0}$ respectively, it can be guaranteed that the two orbits define, by invoking the symmetry of the global system (3)-(6), the half part of a symmetric periodic orbit. If we use the parameterizations $x(u)$ and $x(v)$, respectively, we realize that they are cubic polynomials in their parameter. This fact allows to assure that all the intermediate points are in the same region.

For instance, if we suppose that the orbit in $S_{+}$enters partially the region $S_{0}$, apart from the initial and final point, at least other two points would be in $\Gamma_{+}$and so there should be four roots for the cubic equation $x(u)=1$ with $y_{0}$ and $z_{0}$ given. Obviously this is not possible.

A similar reasoning can be done for the orbit in $S_{0}$ using the parameterization $x(v)$ but here the exclusion of intermediate points out of $S_{0}$ can be guaranteed if we take into account that by letting $T \rightarrow \pm \infty$ (depending on the sign of $\mu$ ) the orbit should arrive at the origin. Thus if there is an excursion into $S_{+}$or $S_{-}$then by considering the extension of the given orbit up to the origin there should be three changes of sign in the derivative of $x(v)$, but this is not allowed for a cubic polynomial.

Proposition 2 allows to establish an equivalence relationship between solutions of equations (11) and symmetric periodic orbits with two points in $\Gamma_{+}$. Once a solution is detected, to study the stability of the corresponding periodic orbit we must locate their characteristic multipliers wit respect to the unit disk of the complex plane. This can be done by using Proposition 3.2 from [20], which assures the similarity between the derivative of the transition map that can be defined from $\Gamma_{+}$to $\Gamma_{-}$corresponding to the half part of the 
symmetric periodic orbit and the product of matrix exponentials $e^{\mathbf{B}_{\mu} T} e^{\mathbf{A} t}$, when evaluated at the appropriate flight times $t$ and $T$. Such transition map is the Poincaré half-return map of the periodic orbit. The above product of matrices has always one eigenvalue equal to -1 , and if $\nu_{1}$ and $\nu_{2}$ are the other two eigenvalues, then $\nu_{1}^{2}$ and $\nu_{2}^{2}$ are the desired characteristic multipliers of the periodic orbit. In [10] it is proved the following simple algebraic result, useful to avoid explicit eigenvalue computations when we have the trace and determinant of the above product of exponential matrices.

Lemma 1. For $p, q \in \mathbb{R}$ the roots of the quadratic $x^{2}-p x+q=0$ are inside the unit circle of the complex plane if and only if the two conditions $|q|<1$ and $|p|<q+1$ are satisfied.

Endowed with these tools, the proof of Theorem 1 can be tackled.

\subsection{Proof of Theorem 1}

In order to show Theorem 1 , we start by considering the easier case $\mu=0$, which corresponds with its statement (d) and needs a special treatment.

\subsubsection{The case $\mu=0$}

For the solution $(x(t), y(t), z(t))$ of system (5)-(6) in the region $S_{+} \cup \Gamma_{+}$ starting at the point $\left(1, y_{0}, z_{0}\right)$ when $t=0$ we can use (7) and (8). The solution $(X(T), Y(T), Z(T))$ of system (4) in the region $\Gamma_{-} \cup S_{0} \cup \Gamma_{+}$passing through the point $\left(1, Y_{0}, Z_{0}\right)$ when $T=0$ is here

$$
\left(\begin{array}{c}
X(T) \\
Y(T) \\
Z(T)
\end{array}\right)=e^{\mathbf{B}_{0} T}\left(\begin{array}{c}
1 \\
Y_{0} \\
Z_{0}
\end{array}\right)=\left(\begin{array}{ccc}
1 & -T & \frac{T^{2}}{2} \\
0 & 1 & -T \\
0 & 0 & 1
\end{array}\right)\left(\begin{array}{c}
1 \\
Y_{0} \\
Z_{0}
\end{array}\right) .
$$


If we start from $\left(1, y_{0}, z_{0}\right)$ with $y_{0}<0$, we see that $\dot{x}=-y_{0}>0$ and so we remain for a time $t$ in the right zone until we arrive at $\left(1, Y_{0}, Z_{0}\right)$, where from (7) with $\mu=0$ we get $\left(0, Y_{0}, Z_{0}\right)^{T}=e^{\mathbf{A} t}\left(0, y_{0}, z_{0}\right)^{T}$. Now, using the flow in the central zone and starting from $\left(1, Y_{0}, Z_{0}\right)$, after a time $T$ we follow the half part of a symmetric periodic orbit if the equations

$$
\begin{aligned}
\left(0, Y_{0}, Z_{0}\right)^{T} & =e^{\mathbf{A} t}\left(0, y_{0}, z_{0}\right)^{T} \\
\left(-1,-y_{0},-z_{0}\right)^{T} & =e^{\mathbf{B}_{0} T}\left(1, Y_{0}, Z_{0}\right)^{T}
\end{aligned}
$$

hold. After eliminating $Y_{0}=-y_{0}-T z_{0}$ and $Z_{0}=-z_{0}$ by using the last two equations, and some standard simplifications we arrive at the algebraic system of equations

$$
\begin{aligned}
& 0=\left(1-4 u+3 u^{2}\right) y_{0}+\left(1-2 u+u^{2}\right) z_{0} \\
& 0=\left(1-u+2 u^{2}\right) y_{0}+\left(T-u+u^{2}\right) z_{0}, \\
& 0=\left(2 u-2 u^{2}\right) y_{0}+\left(1+2 u-u^{2}\right) z_{0}, \\
& 0=4+2 T y_{0}+T^{2} z_{0}
\end{aligned}
$$

where we have used (8) with $u=e^{-t}$. We observe that the first and third equations are homogeneous in $\left(y_{0}, z_{0}\right)$ and do not depend on $T$. The determinant of this homogeneous system of equations must vanish, since from the last equation the values $\left(y_{0}, z_{0}\right)=(0,0)$ are not admissible solutions. Computing such determinant, we must have $(1-u)(1+u)\left(1-4 u+u^{2}\right)=0$. Thus the only meaningful value is $u=2-\sqrt{3}$, because the other do not belong to the interval $(0,1)$. From this moment we assume this value for $u$, removing the third equation and non-vanishing factors, and using the condition 
$1-4 u+u^{2}=0$ to get a simplified system of equations, namely

$$
u y_{0}+z_{0}=0, \quad(7 u-1) y_{0}+(T+3 u-1) z_{0}=0, \quad 4+2 T y_{0}+T^{2} z_{0}=0
$$

Now we get only one solution, as given in the statement (d) of Theorem 1 .

To study the stability of this periodic orbit, we follow the ideas of the end of Section 2.1 and compute the trace and determinant of the matrix $e^{\mathbf{B}_{0} T} e^{\mathbf{A} t}$. We get

$$
\begin{aligned}
-1+\nu_{1}+\nu_{2} & =u+u^{2}+u^{3}-T\left(u+20 u^{2}-21 u^{3}\right) / 2+T^{2}\left(3 u-12 u^{2}+9 u^{3}\right) / 2, \\
-\nu_{1} \nu_{2} & =u^{6}
\end{aligned}
$$

We will apply Lemma 1. It is clear from $q=-u^{6}$ that $|q|<1$. After substituting the values of $T$ and $u$ we see that $p=\nu_{1}+\nu_{2}=-6(26 \sqrt{3}-45)<$ 0 and $1+q=30(26 \sqrt{3}-45)$ so that $|p|=-p<1+q$ clearly holds. Thus $\nu_{1}, \nu_{2}$, and so the multipliers $\nu_{1}^{2}$ and $\nu_{2}^{2}$ of the periodic orbit, are in the unit circle of the complex plane, so that it is hyperbolic and stable. Thus statement (d) of Theorem 1 is shown.

\subsubsection{The case $\mu \neq 0$}

We will analyze the solutions of equations (11) and their relation with the symmetric periodic orbits of system (3)-(6). We first realize by direct inspection that the four equations are linear in $y_{0}$ and $z_{0}$. Furthermore the polynomial $e_{1}$ is cubic in $u$ and independent on $v$, having the trivial factor $u-1$ since $x(0)=1$ by definition. Also the polynomial $e_{2}$ is cubic in $v$ and independent on $u$, while polynomials $e_{3}$ and $e_{4}$ are cubic polynomials both in $u$ and $v$ without mixed terms of the form $u^{m} v^{n}$. 
We also note that a dual approach is possible in writing the closing equations. Thus we can define the solution of system (5) in the region $S_{+} \cup \Gamma_{+}$ backwards in time starting from the point $\left(1, Y_{0}, Z_{0}\right)$ when $t=0$ as

$$
\left(\begin{array}{l}
\bar{x}(t) \\
\bar{y}(t) \\
\bar{z}(t)
\end{array}\right)=\left(\begin{array}{c}
1+\mu^{3} \\
6 \mu\left(1-\mu^{2}\right) \\
11 \mu^{2}(1+\mu)
\end{array}\right)+e^{-\mathbf{A} t}\left[\left(\begin{array}{c}
1 \\
Y_{0} \\
Z_{0}
\end{array}\right)-\left(\begin{array}{c}
1+\mu^{3} \\
6 \mu\left(1-\mu^{2}\right) \\
11 \mu^{2}(1+\mu)
\end{array}\right)\right],
$$

where $e^{-\mathbf{A} t}$ has the same expression that $e^{\mathbf{A} t}$ in (8) if we change $u$ by $U=$ $1 / u=e^{t}$. Then we can take $\bar{y}(t)=y_{0}$ and $\bar{z}(t)=z_{0}$ to write the closing equations in the four unknowns $\left(Y_{0}, Z_{0}, U, V\right)$

$$
\bar{x}(t)-1=0, \quad 1+X(T)=0, \quad \bar{y}(t)+Y(T)=0, \quad \bar{z}(t)+Z(T)=0 .
$$

Here $(X(T), Y(T), Z(T))^{T}=e^{\mathbf{B}_{\mu} T}\left(1, Y_{0}, Z_{0}\right)^{T}$, where the matrix $e^{\mathbf{B}_{\mu} T}$ comes from $e^{-\mathbf{B}_{\mu} T}$ if we change $v$ by $V=1 / v=e^{\mu T}$. Note that for all $t>0$ we will have $U>1$, and if $\mu<0(\mu>0)$ then $0<V<1(V>1)$. We get so the following remark.

Remark 2. It should be emphasized that equations (13) give rise to four equations of the form $e_{i}\left(Y_{0}, Z_{0}, U, V\right)=0$, where the four polynomials $e_{i}$ are exactly the same that in (11). Thus, if for $\mu \neq 0$ there exists a symmetric periodic orbit with two points at $\Gamma^{+}$, namely $\left(1, y_{0}, z_{0}\right)$ and $\left(1, Y_{0}, Z_{0}\right)$, taking a time $t$ in passing from the first point to the second one and a time $T$ in going from the last one to $\left(-1,-y_{0},-z_{0}\right)$, then both $\left(y_{0}, z_{0}, u, v\right)$ and $\left(Y_{0}, Z_{0}, U, V\right)$ are solutions of equations (11), where $U=1 / u=e^{t}$ and $V=1 / v=e^{\mu T}$. Thus every symmetric periodic orbit must be associated to two different solutions of equations (11). 
To simplify a bit equations (11), it is useful to do the translation $\tilde{y}_{0}=$ $y_{0}-6 \mu$ and $\tilde{z}_{0}=z_{0}-11 \mu^{2}$ in order to reallocate the $(y, z)$-origin of the plane $x=1$. Next, by removing the factor $(1-u) / 2$ in the equation $e_{1}=0$, we obtain a simplified first equation $\tilde{e}_{1}=0$, where

$$
\tilde{e}_{1}=2 \mu^{3}(u-1)^{2}+u(1-3 u) \tilde{y}_{0}+u(1-u) \tilde{z}_{0}
$$

The new second equation, after multiplying it by $2 \mu^{2}$ becomes $\tilde{e}_{2}=0$, where

$$
\tilde{e}_{2}=2 \mu^{2}\left(1+3 v-3 v^{2}+v^{3}\right)-\mu v(1-v)(1-3 v) \tilde{y}_{0}+v(1-v)^{2} \tilde{z}_{0}
$$

and to obtain a simplified version of the third equation we define the polynomial $\tilde{e}_{3}=\left[-2 \mu e_{3}+3 \tilde{e}_{2}+\mu(3 u-5) \tilde{e}_{1}\right] / 2$, so that the equation becomes $\tilde{e}_{3}=0$, where

$$
\tilde{e}_{3}=\mu^{4}(1-u)^{2}-3 \mu^{2}\left(1+2 v-v^{2}\right)+\mu\left(u^{2}+v-2 v^{2}\right) \tilde{y}_{0}-v(1-v) \tilde{z}_{0} .
$$

Finally to simplify the fourth equation we define the polynomial $\tilde{e}_{4}=e_{4}+$ $\tilde{e}_{3}-\tilde{e}_{2}+(u-2) \tilde{e}_{1}$ and the equation becomes $\tilde{e}_{4}=0$, where

$$
\tilde{e}_{4}=\mu^{4}(1-u)^{2}+\mu^{3}(1-u)(7-u)+6 \mu^{2}(1+v)+\left[\mu\left(u^{2}-v\right)+u(1+u)\right] \tilde{y}_{0}+(u+v) \tilde{z}_{0} .
$$

Now we use the linearity of above equations in $\tilde{y}_{0}, \tilde{z}_{0}$ to solve two equations for them and substitute in the two remaining equations. For this we select as more convenient the second and third modified equations, obtaining

$$
\tilde{y}_{0}=-\frac{(u-1)^{2}(v-1) \mu^{3}+(v+1)\left(v^{2}-4 v+1\right) \mu}{(v-1)\left(u^{2}+v^{2}\right)}
$$

and a longer expression for $\tilde{z}_{0}$, namely

$$
-\frac{(u-1)^{2}(3 v-1)}{(v-1)\left(u^{2}+v^{2}\right)} \mu^{4}-\frac{v\left(5 v^{4}-16 v^{3}+8 v-1\right)+2 u^{2}\left(v^{3}-3 v^{2}+3 v+1\right)}{(v-1)^{2} v\left(u^{2}+v^{2}\right)} \mu^{2} .
$$


Substituting expressions (14)-(15) in the first and fourth equations and removing the trivial factors $-\mu /(v-1)^{2} v\left(u^{2}+v^{2}\right)$ we obtain two new polynomial equations $f_{1}(u, v)=0$ and $f_{2}(u, v)=0$. We have for $f_{1}(u, v)$ the expression

$$
\begin{aligned}
& (u-1)^{3} u(v-1) v(3 v-1) \mu^{3}+(u-1)^{2}(v-1)^{2} v\left(5 u^{2}-u+2 v^{2}\right) \mu^{2} \\
& +(u-1) u\left[2\left(v^{3}-3 v^{2}+3 v+1\right) u^{2}+v\left(5 v^{4}-16 v^{3}+8 v-1\right)\right] \mu \\
& +u(3 u-1)(v-1) v(v+1)\left(v^{2}-4 v+1\right)
\end{aligned}
$$

while the expression of $f_{2}(u, v)$ is not shown for sake of brevity. In fact, a more convenient alternative is to take instead of $f_{2}$ the polynomial $f_{1}(u, v)+$ $(1-u) f_{2}(u, v)$, which after simplifying by $v$ leads to a new polynomial $\tilde{f}_{2}$. Thus $\tilde{f}_{2}(u, v)$ is

$$
\begin{aligned}
& (u-1)^{3}(v-3)(v-1) v^{2} \mu^{3}-(u-1)^{2}(v-1)^{2}\left(2 u^{2}-v^{2} u+5 v^{2}\right) \mu^{2} \\
& -(u-1)\left[u^{2}\left(v^{4}-8 v^{3}+16 v-5\right)-2 v^{2}\left(v^{3}+3 v^{2}-3 v+1\right)\right] \mu \\
& -(u-3) u^{2}(v-1)(v+1)\left(v^{2}-4 v+1\right) .
\end{aligned}
$$

Our next goal will be to characterize the solutions of the polynomial system formed by the two equations

$$
f_{1}(u, v)=0, \tilde{f}_{2}(u, v)=0 .
$$

From Remark 2, note that if $(u, v)$ are both different from zero and satisfy the polynomial system (18) for a given value of $\mu$, the same is true for the pair $(1 / u, 1 / v)$. Note also that if we take $\tilde{Y}_{0}=Y_{0}-6 \mu$, we can write for it the same expression that for $\tilde{y}_{0}$ in (14) by doing the changes $u \rightarrow U$ and $v \rightarrow V$. Then after using the relations $u=1 / U, v=1 / V$, we get

$$
\tilde{Y}_{0}=-\frac{(u-1)^{2}(v-1) v^{2} \mu^{3}-u^{2}(v+1)\left(v^{2}-4 v+1\right) \mu}{(v-1)\left(u^{2}+v^{2}\right)} .
$$


From Proposition 2 and using (14) and (19) we can obtain more quantitative information about the admissible values of $(u, v)$ corresponding to symmetric periodic orbits having exactly 2 points in the plane $\Gamma_{+}$.

Lemma 2. Consider the next two sets of inequalities

$$
\begin{aligned}
& S_{1}=\left\{\mu<0,0<u<1,1<v<2+\sqrt{3}, \mu^{2}<\frac{(v+1)\left[3-(v-2)^{2}\right]}{(u-1)^{2}(v-1)}\right\}, \\
& S_{2}=\left\{\mu>0,0<u<1,2-\sqrt{3}<v<1, \mu^{2}<\frac{u^{2}(v+1)\left[3-(v-2)^{2}\right]}{(u-1)^{2}(1-v) v^{2}}\right\} .
\end{aligned}
$$

The followings statements hold.

(a) Assume that for a given $\mu \neq 0$ there exists a symmetric periodic orbit of system (3)-(5) having exactly 2 points in the plane $\Gamma_{+}$, then, depending on the sign of $\mu$, the corresponding values of $(u, v)$ satisfy one of the two set of inequalities $S_{1}$ and $S_{2}$.

(b) Conversely, if a pair $(u, v)$ is a solution of (18) with $\mu \neq 0$ satisfying one of the two sets of conditions, then system (3)-(6) has one symmetric periodic orbit having exactly 2 points in the plane $\Gamma_{+}$.

Proof. (a) From the hypothesis and conditions given in Proposition 2 we must have for the periodic orbit that $\tilde{y}_{0}<0$ and $\tilde{Y}_{0}>0$. Taking into account that $v=\exp (-\mu T)$, it is clear that $\mu /(1-v)$ is always positive. Thus from (14) and (19), we arrive, both for positive and negative values of $\mu$, to the two inequalities

$$
\begin{aligned}
(u-1)^{2}(v-1) \mu^{2}+(v+1)\left(v^{2}-4 v+1\right) & <0 \\
(u-1)^{2}(v-1) v^{2} \mu^{2}-u^{2}(v+1)\left(v^{2}-4 v+1\right) & >0 .
\end{aligned}
$$


If $\mu<0$ then $v>1$ and the only possibility to satisfy the first inequality is that $v^{2}-4 v+1=(v-2)^{2}-3<0$, and then the second inequality is automatically fulfilled. When $\mu>0$ we have $0<v<1$, and now the first inequality holds but the second one requires again $v^{2}-4 v+1=(v-2)^{2}-3<0$. Thus statement (a) is true.

(b) Reasoning in a converse way, it is now immediate to obtain under both sets of inequalities that $\tilde{y}_{0}<0$ and $\tilde{Y}_{0}>0$. Then from Proposition 2 we must have the stated symmetric periodic orbit. The lemma follows.

Lemma 2 establishes, under adequate hypotheses, the equivalence between solutions of the polynomial system (18) and symmetric periodic orbits having exactly 2 points in the plane $\Gamma_{+}$. The remaining task is then to find the solutions of the polynomial system (18) satisfying the hypotheses of Lemma 2. In particular we will only need the solutions with $(u, v)$ in the open rectangle $W=(0,1) \times(2-\sqrt{3}, 2+\sqrt{3})$.

We start by computing the resultant polynomial of the elimination of $\mu$ from the equations $f_{1}(u, v)=0$ and $\tilde{f}_{2}(u, v)=0$, getting a long expression in the form $2(1-u)^{6} u(1-v)^{6} v^{4}(1+v)(u+v)\left(u^{2}+v^{2}\right)^{2}\left(1-4 v+v^{2}\right) K(u, v)$, where $K(u, v)$ is

$$
\begin{aligned}
& 8(3 v-1)\left(v^{3}-3 v^{2}+3 v+1\right)^{2} u^{8} \\
& +\left(5 v^{9}+53 v^{8}-235 v^{7}-245 v^{6}+1737 v^{5}-1061 v^{4}-637 v^{3}-179 v^{2}+26 v+24\right) u^{7} \\
& -v\left(169 v^{8}-1114 v^{7}+1100 v^{6}+1474 v^{5}-950 v^{4}-726 v^{3}-1356 v^{2}+398 v-83\right) u^{6} \\
& +v\left(10 v^{10}-170 v^{9}+876 v^{8}+851 v^{7}-4659 v^{6}+1545 v^{5}+1671 v^{4}-683 v^{3}+1137 v^{2}-199 v+5\right) u^{5} \\
& -2 v^{3}\left(55 v^{8}-38 v^{7}-1536 v^{6}-218 v^{5}+4754 v^{4}-218 v^{3}-1536 v^{2}-38 v+55\right) u^{4} \\
& +v^{3}\left(5 v^{10}-199 v^{9}+1137 v^{8}-683 v^{7}+1671 v^{6}+1545 v^{5}-4659 v^{4}+851 v^{3}+876 v^{2}-170 v+10\right) u^{3} \\
& +v^{5}\left(83 v^{8}-398 v^{7}+1356 v^{6}+726 v^{5}+950 v^{4}-1474 v^{3}-1100 v^{2}+1114 v-169\right) u^{2} \\
& +v^{5}\left(24 v^{9}+26 v^{8}-179 v^{7}-637 v^{6}-1061 v^{5}+1737 v^{4}-245 v^{3}-235 v^{2}+53 v+5\right) u \\
& -8(v-3) v^{7}\left(v^{3}+3 v^{2}-3 v+1\right)^{2} .
\end{aligned}
$$


For the definition and the basic properties of the resultant of two polynomials see [21] and [22]. Obviously, we can discard all the previous factors so that we will pay attention only to the roots of the equation $K(u, v)=0$.

Lemma 3. The number of solutions of the equation $K(u, v)=0$ in $W$, that is, with $0<u<1$ and $2-\sqrt{3}<v<2+\sqrt{3}$ is as displayed in Table 1, see also Figure 4. The extremes of intervals with different number of solutions appear in Table 2.

PROOF. In this proof all the computations are based in the root determination of polynomials in only one variable, which is a numerical process that can be made with as much accuracy as needed. In fact, we can select any value of $v=\bar{v}$ in the interval $(2-\sqrt{3}, 2+\sqrt{3})$ and then follow a systematic search for the roots $u \in(0,1)$ of the corresponding polynomial $K(u, \bar{v})$.

It is well-known that polynomial roots are continuous complex functions of the coefficients of the polynomial, excepting when the highest degree coefficient vanishes but in this case one root goes to (comes from) infinity. Thus by varying $\bar{v}$, the number of real roots of $K(u, \bar{v})$ with $u \in(0,1)$ can change only at values of $v$ where the curve $K(u, v)=0$ has a horizontal tangent, a branching point, or one solution escapes through the boundary of the rectangle $W$. We first study possible roots in the boundary of $W$. Starting at the top of the rectangle $W$, the polynomial $K(u, 2+\sqrt{3})$ has $u_{7}$ as the unique solution for $u>0$. This follows easily from the fact that $K(u, 2+\sqrt{3})=$ $288(71+41 \sqrt{3})(2+\sqrt{3}+u)(\delta+u)\left(\delta+2 \delta u+2 u^{2}+u^{3}\right)\left(-\delta+3 \delta u-3 u^{2}+u^{3}\right)$, with $\delta=7+4 \sqrt{3}$, and the last factor is the only one giving positive real roots. The point $P_{7}=\left(u_{7}, v_{7}\right)$ is the top limit endpoint of a branch of solutions of 


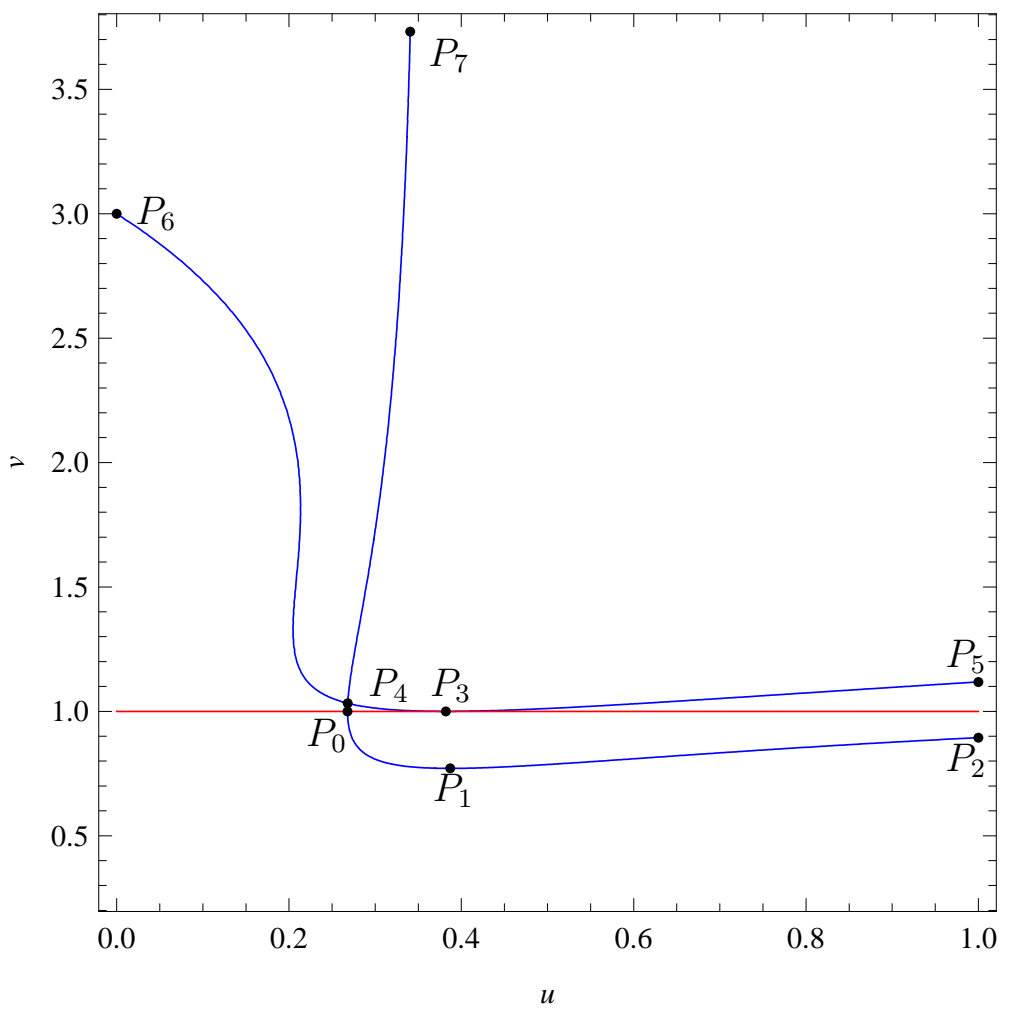

Figure 4: The zero level set of $K(u, v)$ in the rectangle $W=(0,1) \times(2-\sqrt{3}, 2+\sqrt{3})$. The horizontal line drawn is $v=1$. Points $P_{2}, P_{5}, P_{6}$ and $P_{7}$ are in the boundary of the open rectangle.

$K(u, v)=0$ in the open rectangle under study. Since $(2-\sqrt{3})^{-1}=2+\sqrt{3}$ and a direct computation shows that $u^{8} v^{14} K(1 / u, 1 / v)=K(u, v)$, according to Remark 2 , the only positive root for $K(u, 2-\sqrt{3})=0$ is $1 / u_{7}>1$; so this equation has no solution for $u \in(0,1)$.

For the left edge of the rectangle we get

$$
K(0, v)=8(3-v) v^{7}\left(1-3 v+3 v^{2}+v^{3}\right)^{2},
$$

so that the unique point that could be a limit point of an interior branch of 


\begin{tabular}{|c|c|c|}
\hline Range & \# Solutions & \# Periodic orbits \\
\hline$v_{6} \leq v<v_{7}$ & 1 & 1 \\
\hline$v_{5} \leq v<v_{6}$ & 2 & 1 \\
\hline$v_{4}<v<v_{5}$ & 3 & 2 \\
\hline$v=v_{4}$ & 2 & 2 \\
\hline$v_{3}<v<v_{4}$ & 3 & 2 \\
\hline$v=v_{3}$ & 2 & 1 \\
\hline$v_{2}<v<v_{3}$ & 1 & 1 \\
\hline$v_{1}<v<v_{2}$ & 2 & 2 \\
\hline$v=v_{1}$ & 1 & 1 \\
\hline $2-\sqrt{3}<v<v_{1}$ & 0 & 0 \\
\hline
\end{tabular}

Table 1: Number of solutions of $K(u, v)=0$ in the open rectangle $W$. Regarding the last column, we see that not all these solutions correspond with symmetric periodic orbits with two points in $\Gamma^{+}$, as shown in Lemma 4 . 
solutions of $K(u, v)=0$ in the open rectangle is $P_{6}=(0,3)$. In the right edge the situation is more involved as $K(1, v)$ factorizes in a polynomial of degree twelve multiplied by $2(1+v)^{2}$. Such polynomial has two solutions, namely $v_{2}$ and $v_{5}$, see Table 2 , satisfying $v_{2} v_{5}=1$. We also obtain

$$
K(u, 1)=64(1+u)^{2}\left(1-4 u+u^{2}\right)\left(1-3 u+u^{2}\right)^{2}
$$

where the last two factors have solutions with $0<u<1$, namely $u_{0}=2-\sqrt{3}$ and $u_{3}=(3-\sqrt{5}) / 2$, being the last value a double root. In fact, the point $P_{3}$ is a point of horizontal tangent for the curve $K(u, v)=0$.

Now we search for other points belonging to the curve $K(u, v)=0$ with horizontal tangent. Taking derivatives with respect to $u$ in $K(u, v)=0$, we see that $d v / d u=-K_{u} / K_{v}$, so that the wanted points $(u, v) \in W$ must verify

$$
K(u, v)=0, \quad K_{u}(u, v)=0,
$$

with $K_{v}(u, v) \neq 0$. To study the solutions of (20) we compute the resultant with respect to the variable $v$. We obtain a polynomial $R(u)$, which excepting a integer factor, is $(-1+u)^{2} u^{35}(1+u)^{4}\left(1-3 u+u^{2}\right)\left(1+u+u^{2}\right)^{2} R_{26}(u)^{2} R_{62}(u)$. The polynomials $R_{26}$ and $R_{62}$ have the degrees indicated in their subscripts and are not explicitly given for sake of brevity. The factor $1-3 u+u^{2}$ gives again the value $u_{3}$, already obtained before. The other roots of $R(u)$ in $(0,1)$ are approximately $0.041177,0.085276,0.090199,0.252159,0.268219$ and 0.387007. For these values of $u$ we must find the corresponding value of $v$ verifying (20) to guarantee that the corresponding point is in $W$. For instance, when $u=0.041177$ there is no value for $v$ in the range $(2-\sqrt{3}, 2+$ $\sqrt{3}$ ) verifying $(20)$. After checking the other points we conclude that points $P_{1}$ and $P_{3}$ are the unique points of the curve $K(u, v)=0$ with horizontal 


\begin{tabular}{|l|l|}
\hline Value of $u_{i}$ & Value of $v_{i}$ \\
\hline$u_{7}=1-\sqrt[3]{6(5+3 \sqrt{3})}+\sqrt[3]{2(9+5 \sqrt{3})} \approx 0.340721$ & $v_{7}=2+\sqrt{3}$ \\
\hline$u_{6}=0$ & $v_{6}=3$ \\
\hline$u_{5}=1$ & $v_{5} \approx 1.118278$ \\
\hline$u_{4} \approx 0.268219$ & $v_{4} \approx 1.032622$ \\
\hline$u_{3}=(3-\sqrt{5}) / 2 \approx 0.381966$ & $v_{3}=1$ \\
\hline$u_{2}=1$ & $v_{2} \approx 0.894232$ \\
\hline$u_{1} \approx 0.387007$ & $v_{1} \approx 0.770719$ \\
\hline$u_{0}=2-\sqrt{3} \approx 0.267949$ & $v_{0}=1$ \\
\hline
\end{tabular}

Table 2: Numerical values corresponding to marked points in Figure 4.

tangent in $W$, while $P_{4}$ is a branching point, since then both $K_{u}$ and $K_{v}$ vanish.

To show the statement, regarding the first two columns of Table 1, it now suffices to take intermediate values of $\bar{v}$ in the corresponding range and look for the number of solutions of $K(u, \bar{v})$ in $(0,1)$. This is a standard computation for polynomials in one variable and will be not detailed.

Lemma 4. The number of symmetric periodic solutions of system (3)-(6) with two points in $\Gamma^{+}$is as indicated in last column of Table 1.

Moreover the two branches of symmetric periodic solutions with exactly 2 points in the plane $\Gamma_{+}$are as indicated in Theorem 1.

Proof. To guarantee that a solution of equations (3)-(6) corresponds to a symmetric periodic orbit with only two points in $\Gamma^{+}$, we have to apply 
Lemma 2 to all the solutions of Lemma 3. We start by checking the sign of $\mu$ for such solutions.

From eqs. (16) and (17), we see that the common factors of terms of degree zero in $\mu$ are $u(v-1)(v+1)\left(v^{2}-4 v+1\right)$. Thus when one follows a branch of solutions shown in Figure 4, the sign of $\mu$ only can change if one of these factors vanishes. Accordingly, in the rectangle $W$ the value of $\mu$ only can vanish in the line $v=1$. This happens for instance in the point $P_{0}$ with coordinates $\left(u_{0}, v_{0}\right)=(2-\sqrt{3}, 1)$ that corresponds with the only periodic orbit already analyzed for $\mu=0$. However, it must be emphasized that $v-1$ is also a common factor of third degree terms in $\mu$ of eqs. (16) and (17). In fact, it is not difficult to see that if we denote by $\mu(u)$ the value of $\mu$ along the branch of solutions from $P_{3}$ to $P_{5}$ we have $\lim _{u \rightarrow u_{3}^{+}} \mu(u)=-\infty$, and $\lim _{u \rightarrow u_{5}^{-}} \mu(u)=-\infty$. Analogously, it can be shown for the branch from $P_{3}$ to $P_{4}$ that $\lim _{u \rightarrow u_{3}^{-}} \mu(u)=+\infty$, being $\mu$ positive for all the branch from $P_{3}$ to $P_{6}$. Then by applying Lemma 2 we discard this branch for counting periodic solutions.

In short, except the branch from $P_{3}$ to $P_{6}$, the sign of $\mu$ is consistent with Lemma 2. To finish the first statement it remains to show the last inequalities in $S_{1}$ and $S_{2}$, depending on the sign of $\mu$.

To check the last inequality in $S_{1}$ we start by looking for possible values of $(u, v)$ in the branches $P_{0^{-}} P_{7}$ or $P_{3}-P_{5}$ where such inequality is not true any longer, that is

$$
(u-1)^{2}(v-1) \mu^{2}-(v+1)\left[3-(v-2)^{2}\right]=0 .
$$

Using the above expression to simplify (16) and (17) and removing trivial 
non-vanishing factors, we obtain the two conditions

$$
\begin{aligned}
(-1+u) u\left(1+3 v-3 v^{2}+v^{3}\right) \mu-v\left(-1+4 v-4 v^{3}+v^{4}\right) & =0 \\
(-1+u)\left(-5+16 v-8 v^{3}+v^{4}\right) \mu+(-5+u)\left(-1+4 v-4 v^{3}+v^{4}\right) & =0 .
\end{aligned}
$$

Eliminating $\mu$ between these two equations and between the first of them and equation (21), we get from the polynomial resultants, removing again trivial non-vanishing factors, the conditions

$$
\begin{aligned}
& h_{1}(u, v)=(-1+v)^{3} v^{2}(1+v)\left(1-4 v+v^{2}\right)+u^{2}\left(1+3 v-3 v^{2}+v^{3}\right)^{2}=0, \\
& h_{2}(u, v)=u(u-5)\left(1+3 v-3 v^{2}+v^{3}\right)+v\left(-5+16 v-8 v^{3}+v^{4}\right)=0 .
\end{aligned}
$$

Computing now the polynomial resultant of $h_{1}$ and $h_{2}$ with respect to $u$, we obtain a polynomial in $v$ whose only roots in the interval $(1,2+\sqrt{3})$ are $v=2$ and $v \approx 1.267616$. Going back with these values to $h_{1}=0$ and $h_{2}=0$, we obtain the points $(u, v)=(-2,2)$ and $(u, v) \approx(0.205425,1.267616)$. Clearly, only the last point is in $W$ being in fact on the discarded branch $P_{3}-P_{6}$. The conclusion is that, by continuity, the sign of the left hand side of (21) does not change along the branches $P_{0}-P_{7}$ and $P_{3}-P_{5}$.

Since at $P_{0}$ the left hand side of $(21)$ is equal to -4 , we can assure that the last inequality in $S_{1}$ holds for all the points of the branch $P_{0^{-}} P_{7}$. We take now an arbitrary point of the branch $P_{3}-P_{5}$ to check that such inequality is also fulfilled along the branch. We select $u=1 / 2$ and compute the values of $v \approx 1.011567$ and $\mu \approx-23.460760$ for the corresponding point in the branch, so that the value of the left hand side of (21) is approximately -2.477814 . Then we conclude from Lemma 2 that all the points of the branches $P_{0}-P_{7}$ and $P_{3}-P_{5}$ correspond to periodic orbits of system (3)-(6). 
It remains to show that the same is true for the branch $P_{0^{-}} P_{2}$, where the sign of $\mu$ is positive, and now we must check the last inequality in $S_{2}$. We start by looking for possible values of $(u, v)$ in the branch where such inequality is not true any longer, that is

$$
(u-1)^{2}(v-1) v^{2} \mu^{2}-u^{2}(v+1)\left[3-(v-2)^{2}\right]=0 .
$$

Using the above expression to simplify (16) and (17) and removing trivial non-vanishing factors, we now obtain the two conditions

$$
\begin{aligned}
(1-5 u)\left(1-v^{2}\right)\left(1-4 v+v^{2}\right)+\mu(1-u)\left(1-8 v+16 v^{3}-5 v^{4}\right) & =0, \\
u^{2}\left(1-v^{2}\right)\left(1-4 v+v^{2}\right)-\mu(1-u) v^{2}\left(1-3 v+3 v^{2}+v^{3}\right) & =0 .
\end{aligned}
$$

Eliminating $\mu$ between these two equations and between the first of them and equation (22), we get from the polynomial resultants, removing again trivial non-vanishing factors, the conditions

$$
\begin{aligned}
& \tilde{h}_{1}(u, v)=u^{2}(1-v)^{3}(1+v)\left(1-4 v+v^{2}\right)+v^{2}\left(1-3 v+3 v^{2}+v^{3}\right)^{2}=0, \\
& \tilde{h}_{2}(u, v)=(1-5 u) v^{2}\left(1-3 v+3 v^{2}+v^{3}\right)+u^{2}\left(1-8 v+16 v^{3}-5 v^{4}\right)=0 .
\end{aligned}
$$

Computing now the polynomial resultant of $\tilde{h}_{1}$ and $\tilde{h}_{2}$ with respect to $u$, we obtain a polynomial in $v$ whose only roots in the interval $(2-\sqrt{3}, 1)$ are $v=$ $1 / 2$ and $v \approx 0.788883$. Going back with these values to $\tilde{h}_{1}=0$ and $\tilde{h}_{2}=0$, we obtain the points $(u, v)=(-1 / 2,1 / 2)$ and $(u, v) \approx(4.867950,0.788883)$. Clearly, no point is in $W$ and, by continuity, the sign of the left hand side of (22) does not change along the branch $P_{0^{-}} P_{2}$.

Since at $P_{0}$ the left hand side of $(22)$ is equal to $-4(2-\sqrt{3})^{2}$, we can assure that the last inequality in $S_{2}$ holds for all the points of the branch 
$P_{0}-P_{2}$. We then conclude from Lemma 2 that all the points of the branch $P_{0}-P_{2}$ correspond to periodic orbits of system (3)-(6).

Now, to count the number of periodic orbits, we only have to remark that the point $P_{0}$ corresponds with the only periodic orbit detected for $\mu=0$. This explains that in passing such point the number of periodic orbits does not change. Thus the first statement follows.

To show the second statement, we first consider the branch from $P_{3}$ to $P_{5}$. By considering the common factors of the coefficients of third degree in (16) and (17), we conclude that in both ends the value of $\mu$ tends to $-\infty$. By continuity the value of $\mu(u)$ reaches a maximum negative value. This value corresponds with $\mu_{S N 1}$, which can be computed by standard procedures with as much accuracy as desired.

The value of $\mu$ in $P_{1}$ is positive, being also positive for all points from $P_{0}$ to $P_{2}$. Furthermore, reasoning as before, $\lim _{u \rightarrow u_{2}^{-}} \mu(u)=+\infty$. From $P_{0}$ to $P_{7}$, the value of $\mu$ is negative and, considering the common factors of the coefficients of zero degree in (16) and (17), it vanishes in both endpoints. By continuity the value of $\mu$ reaches a minimum negative value corresponding to $\mu_{S N 2}$ and the statement follows.

From Lemma 3 and Lemma 4, Theorem 1 is shown with the exception on the stability assertions about the periodic orbits. It suffices to compute the trace and determinant of the above product of matrix exponentials and apply Lemma 1. After tedious but standard computations, the stability of the periodic orbits can be obtained and the proof of Theorem 1 is complete. 


\section{Computations leading to the first harmonic bifurcation diagram}

Before applying the describing function method to our system (3)-(6), we recall that under their assumptions, if $\sigma(t) \approx \sigma_{1} \sin \omega t$ then $\varphi(\sigma(t)) \approx$ $\sigma_{1} N\left(\sigma_{1}\right) \sin \omega t$, where $N($.$) is the describing function of the normalized satu-$ ration representing the nonlinear gain factor for the first harmonic amplitude, that is $N\left(\sigma_{1}\right)=1$ for $0<\sigma_{1}<1$, and

$$
N\left(\sigma_{1}\right)=\frac{1}{\pi \sigma_{1}} \int_{0}^{2 \pi} \varphi\left(\sigma_{1} \sin \theta\right) \sin \theta d \theta=\frac{2}{\pi}\left(\arcsin \left(\frac{1}{\sigma_{1}}\right)+\frac{1}{\sigma_{1}} \sqrt{1-\frac{1}{\sigma_{1}^{2}}}\right)
$$

for $\sigma_{1} \geq 1$. Thus $N\left(\sigma_{1}\right)$ is decreasing for $\sigma_{1}>1$ and tends to zero when $\sigma_{1} \rightarrow \infty$. Then the pair $\left(\sigma_{1}, \omega\right)$ corresponds with a symmetric periodic orbit of the system when the determining equation

$$
1+N\left(\sigma_{1}\right) G(j \omega)=0
$$

is satisfied. Here $G(s)=\mathbf{c}^{T}(\mathbf{A}-s \mathbf{I})^{-1} \mathbf{b}$ is the transfer function of the system. Effectively, after the formal identification $s=d / d t$ we can write system (1) in the form $(\mathbf{A}-s \mathbf{I}) \mathbf{x}+\mathbf{b} \varphi\left(\mathbf{c}^{T} \mathbf{x}\right)=0$. If we apply it the $1 \times n$ matrix polynomial differential operator $\mathbf{c}^{T} \operatorname{adj}(\mathbf{A}-s \mathbf{I})$, where $\operatorname{adj}(\mathbf{A}-s \mathbf{I})(\mathbf{A}-s \mathbf{I})=\operatorname{det}(\mathbf{A}-s \mathbf{I}) \mathbf{I}$, we see that

$$
\mathbf{c}^{T} \operatorname{adj}(\mathbf{A}-s \mathbf{I})(\mathbf{A}-s \mathbf{I}) \mathbf{x}=\mathbf{c}^{T} \operatorname{det}(\mathbf{A}-s \mathbf{I}) \mathbf{I} \mathbf{x}=\operatorname{det}(\mathbf{A}-s \mathbf{I}) \mathbf{c}^{T} \mathbf{x},
$$

and then we arrive at the scalar differential equation in the output $\sigma$

$$
\operatorname{det}(\mathbf{A}-s \mathbf{I}) \sigma(t)+\mathbf{c}^{T} \operatorname{adj}(\mathbf{A}-s \mathbf{I}) \mathbf{b} \varphi(\sigma(t))=0 .
$$

Thus equation (24) arises by assuming $j \omega$ to be a root of the characteristic polynomial of the scalar linear substitution differential equation that results 
by substituting in (25) the nonlinearity $\varphi(\sigma(t))$ by $N\left(\sigma_{1}\right) \sigma(t)$, see [3, 18] for more details. Note that in our case $\mathbf{c}^{T} \mathbf{x}(t)=x(t)$, so that for every solution pair $\left(\sigma_{1}, \omega\right)$ of $(24)$ the describing function method predicts an elliptic periodic orbit given by

$x(t) \approx \sigma_{1} \sin \omega t$,

$y(t) \approx 6 \sigma_{1}\left[(\mu+1) N\left(\sigma_{1}\right)-1\right] \sin \omega t-\omega \sigma_{1} \cos \omega t$,

$z(t) \approx \sigma_{1}\left[11\left(\mu^{2}-1\right) N\left(\sigma_{1}\right)+11-\omega^{2}\right] \sin \omega t-6 \sigma_{1} \omega\left[(\mu+1) N\left(\sigma_{1}\right)-1\right] \cos \omega t$,

where we have used $\varphi\left(\mathbf{c}^{T} \mathbf{x}(t)\right) \approx \sigma_{1} N\left(\sigma_{1}\right) \sin \omega t$ and (3)-(6).

Typically the determining equation (24) is solved graphically by plotting in the complex plane the curve $G(j \omega)$ for $\omega>0$ and the set corresponding to $-1 / N\left(\sigma_{1}\right)$, which in our case is the interval $(-\infty,-1]$, and looking for possible intersections of both sets. Here we will solve the determining equation (24) in an analytical way as follows. We introduce for any $\sigma_{1} \geq 1$ a negative auxiliary variable $r=r\left(\sigma_{1}\right)=1-1 / N\left(\sigma_{1}\right) \leq 0$, and solve instead of $(24)$ the equivalent equation

$$
r=1+G(j \omega)=\left.\frac{\operatorname{det}\left[s \mathbf{I}-\left(\mathbf{A}+\mathbf{b c}^{T}\right)\right]}{\operatorname{det}(s \mathbf{I}-\mathbf{A})}\right|_{s=j \omega},
$$

where the last equality comes from a corollary of the Schur's lemma, see [2]. Now we give a proof of Proposition 1, and next we describe the computations needed to get $A_{\mathrm{HB}}$, the harmonic balance amplitude approximation for the point $\left(1, y_{0}, z_{0}\right)$, to be compared with the exact value $A_{C}$.

\subsection{Proof of Proposition 1}

Substituting A, b and $\mathbf{c}$ in equation (27), to predict approximately the different periodic orbits, we must look for solutions with $r \leq 0$ and $\omega>0$ of 
the equation

$$
r=\left.\frac{s^{3}-6 \mu s^{2}+11 \mu^{2} s-6 \mu^{3}}{s^{3}+6 s^{2}+11 s+6}\right|_{s=j \omega}=\frac{6 \mu\left(\omega^{2}-\mu^{2}\right)+j \omega\left(11 \mu^{2}-\omega^{2}\right)}{6\left(1-\omega^{2}\right)+j \omega\left(11-\omega^{2}\right)},
$$

or, after separating real and imaginary parts, of the system

$$
r\left(1-\omega^{2}\right)=\mu\left(\omega^{2}-\mu^{2}\right), \quad r \omega\left(11-\omega^{2}\right)=\omega\left(11 \mu^{2}-\omega^{2}\right) .
$$

Eliminating $r$, we arrive at a quadratic in $\Omega=\omega^{2}$, namely

$$
(1+\mu) \Omega^{2}-\left(\mu^{3}+11 \mu^{2}+11 \mu+1\right) \Omega+11 \mu^{2}(1+\mu)=0 .
$$

That is, after eliminating the common factor $(1+\mu)$ we get $\Omega^{2}-\left(\mu^{2}+10 \mu+\right.$ 1) $\Omega+11 \mu^{2}=0$. This equation has positive solutions when $\mu^{2}+10 \mu+1>0$, and the discriminant $\mu^{4}+20 \mu^{3}+58 \mu^{2}+20 \mu+1 \geq 0$. So, we get the two values $\Omega_{ \pm}=\left(\mu^{2}+10 \mu+1 \pm \sqrt{\mu^{4}+20 \mu^{3}+58 \mu^{2}+20 \mu+1}\right) / 2$, whenever the above restrictions are satisfied. These restrictions lead to the conditions $\mu \notin(-5-2 \sqrt{6},-5+2 \sqrt{6}) \approx(-9.898979,-.101021)$ and $\mu \notin(\underline{\mu}, \bar{\mu})$, where

$$
\underline{\mu}=-5-\sqrt{11}-\sqrt{5(7+2 \sqrt{11})}, \quad \bar{\mu}=-5-\sqrt{11}+\sqrt{5(7+2 \sqrt{11})},
$$

which is approximately the interval $(-16.5729,-0.06034)$, so that it is enough to consider only this last one.

We must check if the corresponding values of $r$ are negative. By eliminating $\Omega=\omega^{2}$ in $(28)$ we arrive at $10 r^{2}-\left(\mu^{3}+11 \mu^{2}-11 \mu-1\right) r-10 \mu^{3}=0$, with roots

$$
r_{ \pm}=\frac{(\mu-1)\left(\mu^{2}+12 \mu+1\right) \pm|\mu+1| \sqrt{\mu^{4}+20 \mu^{3}+58 \mu^{2}+20 \mu+1}}{20} .
$$

The expression under the square root is the same than for $\Omega_{ \pm}$. Thus for $\mu<0$ both roots are negative provided that $\mu^{2}+2(5+\sqrt{11}) \mu+1>0$, (this 
also implies $\mu^{2}+12 \mu+1>0$ and $\left.\mu^{2}+2(5-\sqrt{11}) \mu+1>0\right)$ that is for $\mu \notin(\underline{\mu}, \bar{\mu})$. However, for $\mu=0$ the root $r_{+}$vanishes, and for $\mu>0$ only the root $r_{-}$turns out to be negative. Note that the vanishing of $r$ should be related with $N\left(\sigma_{1}\right)=1$, that is we have for the amplitude $\sigma_{1}=1$.

In short the harmonic balance method predict two periodic orbits for $\mu<\underline{\mu}$ and for $\bar{\mu}<\mu<0$. Consequently the analysis predicts saddle-node bifurcations of periodic orbits at such two negative values of $\mu$. At $\mu=0$, it is also predicted the disappearance of one periodic orbit (in some kind of heteroclinic connection, since then $\left.\Omega_{-}=0\right)$. For $\mu>0$, only one periodic orbit remains, namely the associated to the pair $\left(r_{-}, \Omega_{+}\right)$.

The stability of the predicted limit cycles can be deduced in several ways, see [17] or [18]. For the symmetric periodic orbits predicted by harmonic balance in $\mathbb{R}^{3}$, the notions of axial stability and radial stability are useful, see [18]. Roughly speaking, the axial stability is associated to the third root $\rho$ of the characteristic polynomial corresponding to the linear substitution problem for every solution $\left(\sigma_{1}, \omega\right)$ of the determining equation. Such polynomial is in our case

$$
\operatorname{det}(s \mathbf{I}-\mathbf{A})+N\left(\sigma_{1}\right)\left[\operatorname{det}\left(s \mathbf{I}-\mathbf{B}_{\mu}\right)-\operatorname{det}(s \mathbf{I}-\mathbf{A})\right]
$$

which for every solution $\left(\sigma_{1}, \omega\right)$ of the determining equation has the roots $\pm j \omega$ and $\rho$. Thus we can deduce easily from the coefficient of $s^{2}$ in

$$
s^{3}+6 s^{2}+11 s+6-\frac{1}{1-r}\left[6(\mu+1) s^{2}+11\left(\mu^{2}-1\right) s+6\left(\mu^{3}+1\right)\right]
$$

that $\rho=6(\mu+r) /(1-r)$. Since $r$ is negative by definition, $\operatorname{sign}(\rho)=$ $\operatorname{sign}(\mu+r)$ and the axial stability can be guaranteed always for $\mu<0$. 
However for $\mu>0$ the axial stability can be lost. In fact from (30) and using $r_{-}$, we can write

$$
\mu+r_{-}=\frac{2 \mu(\mu+1)(\mu-1)}{\mu^{2}+10 \mu-1+\sqrt{\mu^{4}+20 \mu^{3}+58 \mu^{2}+20 \mu+1}},
$$

and this expression becomes positive for $\mu>1$. Therefore the predicted symmetric periodic orbit for $\mu>1$ is unstable.

The radial stability can be characterized by computing for every solution of the determining equation the sign of the following expression:

$\frac{d}{d \omega} \operatorname{Im} G(j \omega)=\frac{d}{d \omega} \operatorname{Im}[1+G(j \omega)]=\frac{d}{d \omega} \operatorname{Im}\left(\frac{6 \mu\left(\omega^{2}-\mu^{2}\right)+j \omega\left(11 \mu^{2}-\omega^{2}\right)}{6\left(1-\omega^{2}\right)+j \omega\left(11-\omega^{2}\right)}\right)$,

and it is required to have positive sign for stable cases. This is equivalent to the so-called Loeb criterion, see [17]. We obtain so for $(d / d \omega) \operatorname{Im} G(j \omega)$ the expression

$$
\frac{d}{d \omega}\left(6 \omega \frac{\left(1-\omega^{2}\right)\left(11 \mu^{2}-\omega^{2}\right)-\mu\left(\omega^{2}-\mu^{2}\right)\left(11-\omega^{2}\right)}{36\left(1-\omega^{2}\right)^{2}+\omega^{2}\left(11-\omega^{2}\right)^{2}}\right)=\frac{d}{d \omega}\left(6 \omega \frac{\operatorname{num}(\omega)}{\operatorname{den}(\omega)}\right)
$$

where the numerator $\operatorname{num}(\omega)$ is exactly the left hand side of equation (29) determining the value of $\omega$ for the periodic orbit. Denoting such value for $\omega_{*}$ and using that $\operatorname{num}\left(\omega_{*}\right)=0$, we see that

$$
\left.\frac{d}{d \omega}\left(6 \omega \frac{\operatorname{num}(\omega)}{\operatorname{den}(\omega)}\right)\right|_{\omega=\omega_{*}}=\left.6 \omega_{*} \frac{d}{d \omega}\left(\frac{\operatorname{num}(\omega)}{\operatorname{den}(\omega)}\right)\right|_{\omega=\omega_{*}}=\frac{6 \omega_{*} \operatorname{num}^{\prime}\left(\omega_{*}\right)}{\operatorname{den}\left(\omega_{*}\right)} .
$$

Finally we compute $\operatorname{num}^{\prime}(\omega)=2(1+\mu) \omega\left[2 \omega^{2}-\left(\mu^{2}+10 \mu+1\right)\right]$, and recalling the expression for $\Omega_{ \pm}$and the stability criterion, we deduce that for the different values of $\mu$ only one periodic orbit is radially stable, namely the corresponding to $\Omega_{-}$when $\mu<\underline{\mu} \approx-16.572910$, and the corresponding to $\Omega_{+}$when $\mu>\bar{\mu} \approx-0.060339$ (the one that persists for $\mu>0$ ).

The proposition is shown. 


\subsection{Computation of $A_{H B}$}

For the computation of $A_{\mathrm{HB}}$ it suffices to use the approximation to the point $\left(1, y_{H B}, z_{H B}\right)$ given by $(26)$ when we assume $x=1$ and $\dot{x}>0$, that is $\sin \omega t=1 / \sigma_{1}$ and $\cos \omega t=\sqrt{1-1 / \sigma_{1}^{2}}$. Thus

$$
\begin{aligned}
& y_{H B} \approx 6\left[(\mu+1) N\left(\sigma_{1}\right)-1\right]-\omega \sigma_{1} \sqrt{1-1 / \sigma_{1}^{2}}, \\
& z_{H B} \approx 11\left(\mu^{2}-1\right) N\left(\sigma_{1}\right)+11-\omega^{2}-6 \sigma_{1} \omega\left[(\mu+1) N\left(\sigma_{1}\right)-1\right] \sqrt{1-1 / \sigma_{1}^{2}} .
\end{aligned}
$$

To obtain the value of $\sigma_{1}$ from the value of $r$ given in (30), which involves basically the inversion of function $N\left(\sigma_{1}\right)$, it turns out more convenient to work with the saturation angle $\theta$ satisfying $\sigma_{1} \sin (\theta)=1$ with $\theta \in(0, \pi / 2]$. Thus we write

$$
\frac{1}{1-r}=N\left(\sigma_{1}\right)=\frac{2}{\pi}(\theta+\sin \theta \cos \theta) .
$$

Then, after solving numerically the above equation for $\theta$, we get finally some expressions independent on $\sigma_{1}$, namely

$$
\begin{aligned}
& y_{H B} \approx 6 \frac{\mu+r}{1-r}-\omega \frac{\cos \theta}{\sin \theta} \\
& z_{H B} \approx 11 \frac{\mu^{2}-r}{1-r}-\omega^{2}-6 \omega \frac{(\mu+r) \cos \theta}{(1-r) \sin \theta}
\end{aligned}
$$

\section{Realization in the Chua's circuit}

We will see that system (3)-(6) is realized by certain set of parameters in the celebrated Chua's oscillator, see [11] and references therein. The dimensionless equations of the Chua's oscillator are

$$
\begin{aligned}
& \dot{X}=\alpha[Y-b X+(b-a) \operatorname{sat}(X)] \\
& \dot{Y}=X-Y+Z \\
& \dot{Z}=-\beta Y-\gamma Z .
\end{aligned}
$$




\begin{tabular}{|c|c|c|c|c|c|c|}
\hline$i$ & $\alpha_{i}$ & $\beta_{i}$ & $\gamma_{i}$ & $a_{i}$ & $b_{i}$ & $\mu_{i}$ \\
\hline 1 & 0.0998711 & 0.0536638 & 2.04857 & 9.29757 & 29.5524 & -0.662854 \\
\hline 2 & 0.159613 & 1.26329 & 3.66348 & 28.8122 & 8.37350 & -1.54372 \\
\hline 3 & -2.64515 & 0.213248 & 0.736936 & 0.775919 & -1.61166 & 0.0525804 \\
\hline
\end{tabular}

Table 3: Numerical values of parameters where Chua's circuit realizes system (3)-(6).

See [23] or again [11] for the meaning of different parameters. This system can be written in the form used for system (3)-(6) by the linear change of variables

$$
X=x, \quad \alpha Y=-(1+\gamma) x-y, \quad \alpha Z=\left(\gamma^{2}-\beta\right) x+\gamma y+z
$$

well defined for $\alpha \neq 0$.

To show that this oscillator realizes system (3)-(6) it suffices to see that the equality between the linear invariants of the corresponding matrices is possible, namely that the equations

$$
\begin{aligned}
6 \mu & =-1-a \alpha-\gamma, \\
11 \mu^{2} & =-\alpha+\beta+\gamma+a \alpha(1+\gamma), \\
6 \mu^{3} & =\alpha \gamma-a \alpha(\beta+\gamma), \\
-6 & =-1-b \alpha-\gamma, \\
11 & =-\alpha+\beta+\gamma+b \alpha(1+\gamma), \\
-6 & =\alpha \gamma-b \alpha(\beta+\gamma),
\end{aligned}
$$

are solvable. We can assume $a \neq b$ and $\mu \neq-1$ to exclude the purely linear cases. Instead of equations (36)-(38) we will use their respective differences 
with equations (33)-(35), namely

$$
\begin{aligned}
6(\mu+1) & =(b-a) \alpha, \\
11\left(\mu^{2}-1\right) & =(a-b)(1+\gamma) \alpha, \\
6\left(\mu^{3}+1\right) & =(b-a)(\beta+\gamma) \alpha,
\end{aligned}
$$

and assume in the sequel $\alpha \neq 0$. From the division between (40) and (39), we get $\gamma=(5-11 \mu) / 6$, and then by dividing (41) and (39) we obtain $\beta=\left(6 \mu^{2}+5 \mu+1\right) / 6$. Since from (39) we can write $b=a+6(\mu+1) / \alpha$, it suffices to bring these expressions to equations (33)-(35) and solve for variables $a, \alpha$ and $\mu$. Now from (33) we get $a \alpha=-(11+25 \mu) / 6$ and then from (34) we obtain $\alpha=-5\left(17 \mu^{2}+38 \mu+17\right) / 36$. Finally by substituting in (35) we get the cubic $539 \mu^{3}+1161 \mu^{2}+489 \mu-29=0$, which has three real roots $\mu_{i}, i=1,3$. In short we have shown that the Chua's circuit realizes system (3)-(6) in the three different set of dimensionless parameters of Table 3.

From the application of Theorem 1 we now deduce that in the first two cases we must expect global asymptotic stability for the origin, while in the third case, as $0<\mu_{3}<\mu^{*}$, the origin is a repeller and there exists a stable limit cycle. In the last case, by using (18) we get $(u, v) \approx$ $(0.273356,0.891417)$ and then the flight times $t=-\log u \approx 1.29698$ and $T=-(\log v) / \mu \approx 2.18605$. Furthermore from (14)-(15), we can assure that such Chua's oscillator has one stable periodic orbit passing through the point $\left(1, y_{0}, z_{0}\right)=(1,-1.55048,0.491692)$.

\section{References}

[1] D.P. Atherton, Nonlinear control engineering (Describing function anal- 
ysis and design), 2nd edition, Van Nostrand Reinhold, New York 1982.

[2] S. Barnett and R.G. Cameron, Introduction to mathematical control theory, 2nd edition, Clarendon Press, Oxford 1985.

[3] H.K. Khalil, Nonlinear systems, Macmillan, New York 1992.

[4] A.I. Mees, Dynamics of feedback systems, John Wiley \& Sons 1981.

[5] M. Vidyasagar, Nonlinear systems analysis, 2nd ed., Prentice-Hall 1993.

[6] R. Lozano, B. Brogliato, D. Egeland and B. Maschke, Dissipative Systems, Analysis and Control, Theory and Applications, Springer, London, 2000.

[7] N. E. Barabanov, On the Kalman problem, Siberian Math. J. 29 (1988), $333-341$.

[8] J. Bernat and J. Llibre, Counterexample to Kalman and Markus-Yamabe conjectures in dimension larger than 3, Dynamics of Continuous, Discrete and Impulsive Systems 2 (1996), 337-379.

[9] I. Moreno and R. Suárez, Existence of periodic orbits of stable saturated systems, Systems Control Lett. 51 (2004), 293-309.

[10] E. Ponce and J. Ros, On periodic orbits of 3D symmetric piecewise linear systems with real triple eigenvalues, Int. J. Bifurcations and Chaos, 19 (2009), 2391-2399. 
[11] M. P. Kennedy, Three Steps to Chaos-Part II: A Chua's Circuit Premier, IEEE Transactions on Circuits and Systems - I: Fundamental Theory and Applications 40 (1993), 657-674.

[12] A.A. Andronov, A.A. Vitt and S.E. Khaikin, Theory of Oscillators, Pergamon Press, Oxford 1966.

[13] L. Liu, Y.S. Wong and B.H.K. Lee, Nonlinear aeroelastic analysis using the point transformation method, part I: freeplay model, J. Sound and Vibration 253 (2002), 447-469.

[14] D.J. Allwright, Harmonic balance and the Hopf bifurcation theorem, Maths. Proc. Cambridge Phil. Soc. 82 (1977), 453-467.

[15] B. Aguirre, J. Alvarez-Ramirez, G. Fernandez and R. Suarez, First Harmonic Analysis of Linear Control Systems with High-Gain Saturating Feedback, Int. J. Bifurcation and Chaos 7 (1997), 2501-2510.

[16] J. Alvarez and L. E. Curiel, First Harmonic Analysis of Linear Control Systems with High-Gain Saturating Feedback, Int. J. Bifurcation and Chaos, Vol. 7 (1997) No. 8, 1811-1822.

[17] M. Basso and R. Genesio, Analysis and control of limit cycle bifurcations, in G. Chen, D.J. Hill and X. Yu (Eds.): Bifurcation Control, LNCIS 293 (2003), 127-154, Springer, Berlin.

[18] J. Llibre and E. Ponce, Global first harmonic bifurcation diagram for odd piecewise linear control systems, Dynam. Stability Systems 11 (1996), $49-88$. 
[19] P.E. Rapp and A.I. Mees, Spurius prediction of limit cycles in a nonlinear feedback system by the describing function method, Int. J. Control 26 (1977), 821-829.

[20] E. Freire, E. Ponce and J. Ros, The Focus-Center-Limit Cycle Bifurcation in Symmetric 3D Piecewise Linear Systems, SIAM J. Appl. Math. 65 (2005), 1933-1951.

[21] S. Lang, Algebra, 3rd. Edition, Addison-Wesley, 1993.

[22] P. Olver, Classical invariant theory, London Math. Soc. Student Texts, Vol. 44, Cambbridge Univ. Press, New York, 1999.

[23] V. Carmona, E. Freire, E. Ponce, J. Ros and F. Torres, Limit cycle bifurcation in $3 D$ continuous piecewise linear systems with two zones. Application to Chua's circuit, International Journal Bifurcation and Chaos, $\mathbf{1 5}$ (2005), 2469-2484. 